\title{
FIRST RESULTS AND ASPECTS OF IN VIVO DOSIMETRY SYSTEM IMPLEMENTATION OF EXTERNAL RADIATION THERAPY IN TOMSK REGIONAL ONCOLOGY CENTER
}

\author{
Andrey V. Vertinskiy ${ }^{1,2^{*}}$, Leonid G. Sukhikh ${ }^{2}$, Evgeniia S. Sukhikh ${ }^{1,2}$, Yana N. Sutygina ${ }^{1,2}$ \\ ${ }^{1}$ Tomsk Regional Oncology Center, Tomsk, Russia \\ ${ }^{2}$ National Research Tomsk Polytechnic University, Tomsk, Russia
}

\begin{abstract}
This article describes some aspects of the implementation of the system in vivo dosimetry PerFraction with the linear accelerator Elekta Synergy Platform. The first results of in vivo dosimetry application are presented in comparison with the results of $3 D$ phantom-based ArcCHECK dosimetry.
\end{abstract}

Keywords: In vivo dosimetry, portal dosimetry, external beam radiation therapy, PerFraction

\section{INTRODUCTION}

The main purpose of modern radiation therapy is to increase the contrast between the irradiation of the tumor and risk organs. For this purpose, methods of dose delivery with photon radiation intensity modulation (volumetric modulated arc therapy (VMAT), intensity-modulated radiation therapy (IMRT)) are widely introduced into clinical practice. These techniques are characterized by the creation of radiation fields of a complex variable form during the irradiation. The creation of plans with a large dose gradient allows to implement the hypofractionated mode in which the dose per fraction can be up to $15 \mathrm{~Gy}$.

The implementation of complex plans requires the quality assurance (QA) of radiation, which is provided by the implementation of two types of procedures: QA before therapy and QA in the process of irradiation of patients (in-vivo dosimetry).

The development of pre-treatment verification began with $1 \mathrm{D}$ measurement - absolute dose value measurement at one point in a solid phantom, for example, in the isocenter or in critical organs. The next step was $2 \mathrm{D}$ verification, which included the use of film dosimeters and matrix detectors, which allowed obtaining the dose distribution on the plane. A further development was the phantoms for the verification of $3 \mathrm{D}$, allowed to overlay the measured phantom dose distribution on the geometry of the patient (Delta4 (ScandiDos AB, Uppsala, Sweden), ArcCHECK (Sun Nuclear Corp., Melbourne, USA)). This step made it possible to move from gamma index calculation to dose-volume histogram (DVH)-based plan quality assessment [1].

Despite of the importance of QA before irradiation, there are still questions about the quality of reproduction of the radiation plan from fraction to fraction taking into account both the possible inaccuracies and the changes in the anatomical features of the patient during treatment (include changes in weight, size and shape of the tumor, the movement of organs).

In vivo dosimetry methods are used to obtain this information. Their development began from the measurement of dose at the point (e.g. diodes, transistors, scintillation detectors, etc.) to the application of portal dosimetry based on an electronic portal imaging device (EPID) [2]. Initially, EPID was used for $2 \mathrm{D}$ forward dosimetry, which consists of a direct comparison of the image obtained during irradiation with the image expected on the basis of treatment planning system (TPS) calculation. In further development, the resulting portal images were used as the source data for back-projection models to reconstruct the $3 \mathrm{D}$ dose distribution in patients. It is possible to obtain the actual dose distribution in the patient's body and DVHs.

The efficiency of EPID dosimetry has been investigated in several papers and has been highly evaluated [3-8]. Since all modern linear accelerators are equipped with an EPID panel, in vivo dosimetry based on portal images is a quite affordable method with the acceptable accuracy of detection of irradiation errors.

The purpose of this article is to present the results of PerFraction implementation, to highlight some moments that we are faced during adjustment and calibration and to compare the results of $3 \mathrm{D}$ verification with the ArcCHECK verification that is commonly used in our clinic as a standard.

\footnotetext{
*a.v.vertinsky@tomonco.ru
} 


\section{MATERIALS AND METHODS}

\subsection{Description of PerFraction}

The PerFraction (PF) system is an automatic complex (a part of SunCHECK platform) for monitoring the irradiation quality of each fraction delivered to a particular patient. The system is a software installed on a dedicated server that collects information about the treatment sessions (log files of the linear accelerator, Cone beam computed tomography (CBCT) images, portal images of the EPID panel). Based on this information, the built-in Sun Nuclear Dose Calculator (SDC) - graphics processing unit(GPU)-accelerated Collapse Cone Convolution/Superposition (CCC) algorithm performs an independent calculation of the dose distribution in the patient [9-10]. This algorithm, by default, uses a beam model created by Sun Nuclear Corporation, based on the average dose characteristics of five accelerators of the same class.

The dosimetric plan developed by the TPS in the DICOM format is transferred to the PerFraction server. The data includes a set of CT images, a set of structures and individual dose characteristics of the beams. After receiving incoming data the DoseCHECK module (a part of the SunCHECK platform that is used for pretreatment verification) automatically provides independent quality assessment of the planning system by recalculating beam doses using CCC-algorithm. The results are presented as a comparison of Planned (by TPS) and Calculated (QA) doses by point dose and $3 \mathrm{D}$ dose distribution in the structures of the patient.

Pre-treatment QA is made without the presence of a phantom (zero-fraction). The treatment plan is fully reproduced with the collection of EPID-images. The obtained images are analyzed by the gamma index method compared to the "expected" ones and are used to form a $3 \mathrm{D}$ dose distribution.

In vivo dosimetry of each fraction uses daily CBCT and EPID images of the patient in combination with the accelerator log files. All EPID images obtained at the first fraction are selected as a base (baseline). All subsequent images are automatically compared to the baseline. The result of the comparison is an output in the form of DVH distribution structures of the patient [11].

\subsection{Implementation of PerFraction}

It was necessary to carry out a number of procedures, including the configuration of the network parameters of PF server, access to the databases of the linear accelerator, MOSAIQ and the calibration of the EPID panel to make the PF fully operational.
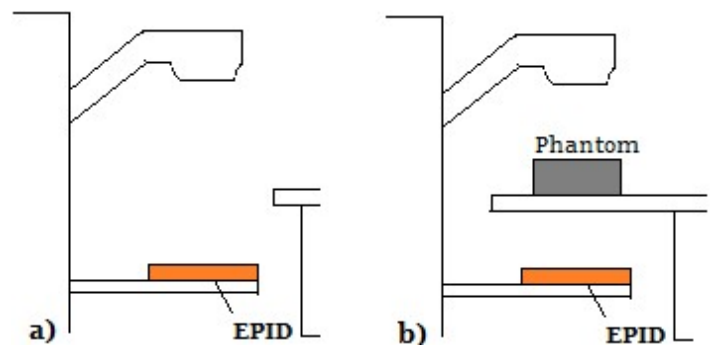

Figure 1. Realization scheme: a) Fraction Zero Absolute Dose calibration without the presence of a table, b) Fraction $\mathrm{N}$ Absolute Dose calibration in the presence of a phantom of a different thickness

Calibration of the EPID panel was held in two stages with standard manufacture procedures [11]. The first stage involved summing up a series of 37 rectangular fields of varying geometry, which are generated by PerFraction software according to the linac model, delivered initially in the air with a fully extended therapeutic table so that the radiation fell directly onto the EPID ("Fraction Zero Absolute Calibration", FZAD) (Figure 1a). The distance from linac source to EPID panel for Elekta Synergy accelerator is $160 \mathrm{~cm}$. Calibration for in vivo transit dosimetry ("Fraction N Absolute Calibration", FNAD) was produced in the second stage, in which the irradiation of a series of fields was carried out in the presence of the solid phantom of different thickness (30 cm, $10 \mathrm{~cm}$ and $\mathrm{o} \mathrm{cm}$ ) (Figure $1 \mathrm{~b}$ ).

After collecting of required data, PerFraction automatically calculate signal-to-dose conversion factors separately for Zero and $\mathrm{N}$-fractions that transform EPID images to dose. The EPID panel calibration procedures were performed for each photon energy used (6 MV and $10 \mathrm{MV}$ ).

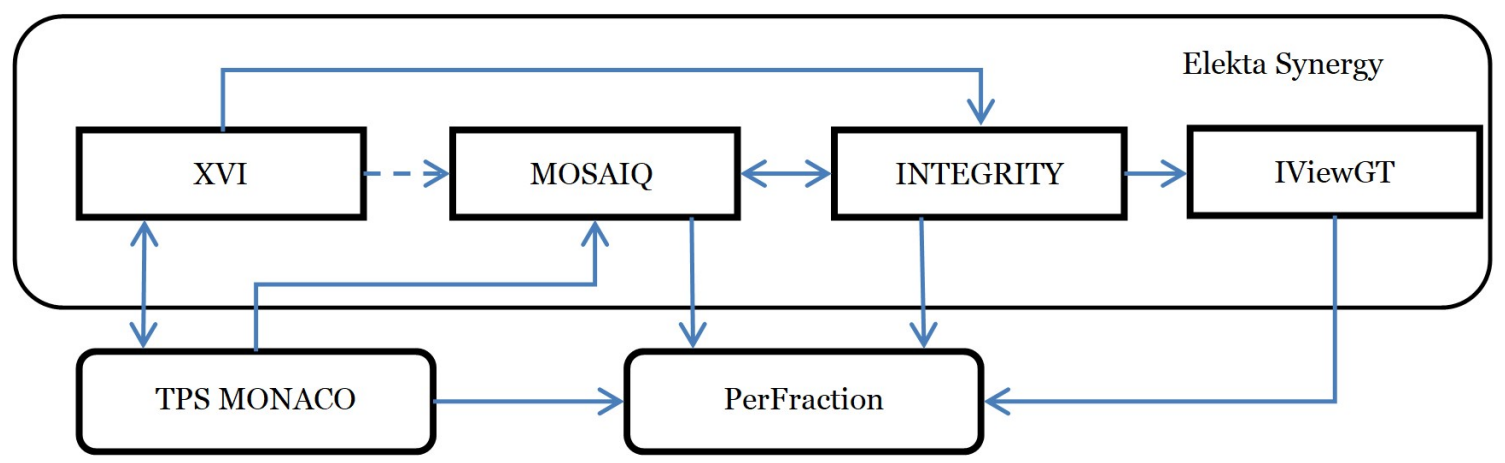

Figure 2. The scheme of interaction between TPS, accelerator elements and PerFraction server

Figure 2 shows the scheme of interaction between the PerFraction server and the accelerator's control stations. From the TPS Monaco, the approved treatment plan with CT-images, structures and beam 
doses is transferred to the XVI system (CBCT), MOSAIQ and PerFraction. The MOSAIQ database exports to INTEGRITY the patient's treatment beams information and stores on the database server the data about the delivered fractions. The XVI station calculates the couch offset to align the plan isocenter with the linac isocenter and transfers the offsets to INTEGRITY. The obtained CBCT images are stored in the XVI station. These images could be transmitted to TPS or MOSAIQ in the DICOM format. The IViewGT station by iCOM link receives the loaded beam's information from INTEGRITY and collects the photon fluence data from linac as EPID images. The PerFraction collects the linac log-files from INTEGRITY, delivered fraction's information and CBCT-images from MOSAIQ, EPID images from the IViewGT station. PerFraction has no software opportunity to retrieve any data from the XVI station. The dotted line indicates the process of files transferring from XVI to MOSAIQ that we had to set up. In our linac configuration, the XVI offsets, which are necessary for PerFraction, do not automatically transfer to MOSAIQ server. Therefore, we had to merge the XVI images with the reference CT images in MOSAIQ directly.

\subsection{Linear accelerator and VMAT plans}

In this study, four plans of external radiation therapy with the VMAT of a different localization and fractionation regimes were used (Table 1). The plans were simulated using the Monaco v 5.02 TPS (Elekta $\mathrm{AB}$, Stockholm, Sweden) using photon beams with energy equal to $6 \mathrm{MV}$ and $10 \mathrm{MV}$ of Elekta Synergy linac. The linear accelerator is equipped with XVI.4.5 and IViewGT v3.4 stations.

Table 1. Localizations and fractionations

\begin{tabular}{|l|l|c|c|}
\hline № & Localization & Total dose, Gy & Fraction dose, Gy \\
\hline 1 & Brain & 60 & 2 \\
\hline 2 & Head\&Neck & 50 & 2 \\
\hline 3 & Prostate & 36.25 & 7.25 \\
\hline 4 & Lymphoma & 36 & 2 \\
\hline
\end{tabular}

Each plan, according to the established internal quality assurance Protocol, has been verified using the ArcCHECK dosimetry system with $3 \mathrm{DVH}$ software (Sun Nuclear Corp. Melbourne, USA). ArcCHECK is a cylindrical phantom with a spiral position of detectors, specially created for the verification of rotational irradiation. $3 \mathrm{DVH}$ software calculates the $3 \mathrm{D}$ dose perturbations arising due to differences between the measured and the planned dose distributions. The calculation result is displayed as a DVH histogram of the reference (TPS) and compared distribution, as well as the total and differentiated (for each structure) gamma index.

The criteria for the evaluation of results were the dose at the point (isocenter) and the DVH statistics of the dose distribution. The percentage discrepancy between the dosimetric parameters for the target (PTV) and organ at risk (OAR) was calculated according to Equation 1, where D(TPS) is the planned dose, D(QA) is the measured dose.

$$
\Delta=\frac{D(T P S)-D(Q A)}{D(T P S)} * 100
$$

\section{RESULTS AND DISCUSSION}

\subsection{Implementation of PerFraction}

The standard calibration procedure of the EPID panels is conducted in accordance with the regulation of service (Las Vegas test). However, during the PerFraction implementation, it was necessary to carry out additional calibration procedures in the IViewGT (for example: dark field, flood field and multi-gain calibration for each energy). As a result, the images of fluence from the EPID panel became more consistent with the expected (Figure 3) and gamma indices of FZAD and FNAD calibrations increased (Table 2).

Table 2. Coincidence of expected and measured calibration fields at the gamma index $y(3 \% / 3 \mathrm{~mm}, \mathrm{GN})$ before and after additional EPID calibration.

\begin{tabular}{|l|l|l|}
\hline PF procedure & before & after \\
\hline FZAD Calibration 6MV & $99.02 \%$ & $99.60 \%$ \\
\hline FZAD Calibration 10MV & $93.1 \%$ & $99.01 \%$ \\
\hline FNAD Calibration 6 MV & $98.60 \%$ & $98.89 \%$ \\
\hline FNAD Calibration 10MV & $94.42 \%$ & $99.25 \%$ \\
\hline
\end{tabular}

\subsection{Verification of VMAT plans}

The results of the DoseCHECK and $3 \mathrm{DVH}$ comparison are shown in Table 3. One can see that 3DVH QA doses for most structures are higher than TPS. For DoseCHECK the situation is different, QA dose is less than the prescribed one. The difference is as high as $7.8 \%$.

The EPID-based in vivo dosimetry result is presented on the example of one fraction dosimetry for the prostate case therapy (Figure 4). The result of the gamma analysis shows the complete convergence of the two distributions by the criterion $\mathrm{y}(3 \% / 3 \mathrm{~mm}, \mathrm{GN})$. DVH statistic of this fraction is presented in Figure 5. As one can see, the maximum doses to the structures obtained by in vivo dosimetry are smaller than DoseCHECK results (at the same time D95(PTV) QA is slightly better hen TPS) and QA doses are closer to TPS doses.

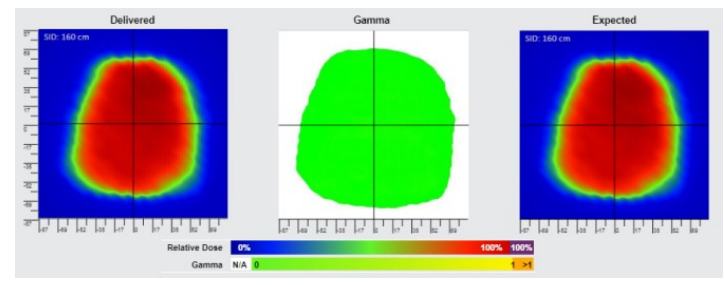

Figure 4. The comparison of delivered EPID (left) and expected (right) doses by the gamma analysis (central image).

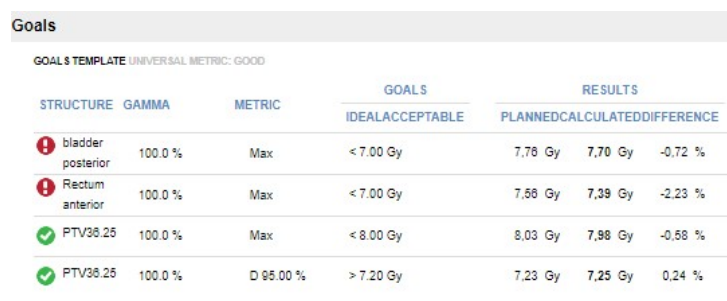

Figure 5. The PerFraction event report with comparison of QA and TPS doses by the EPID-based in vivo dosimetry of prostate case fraction 


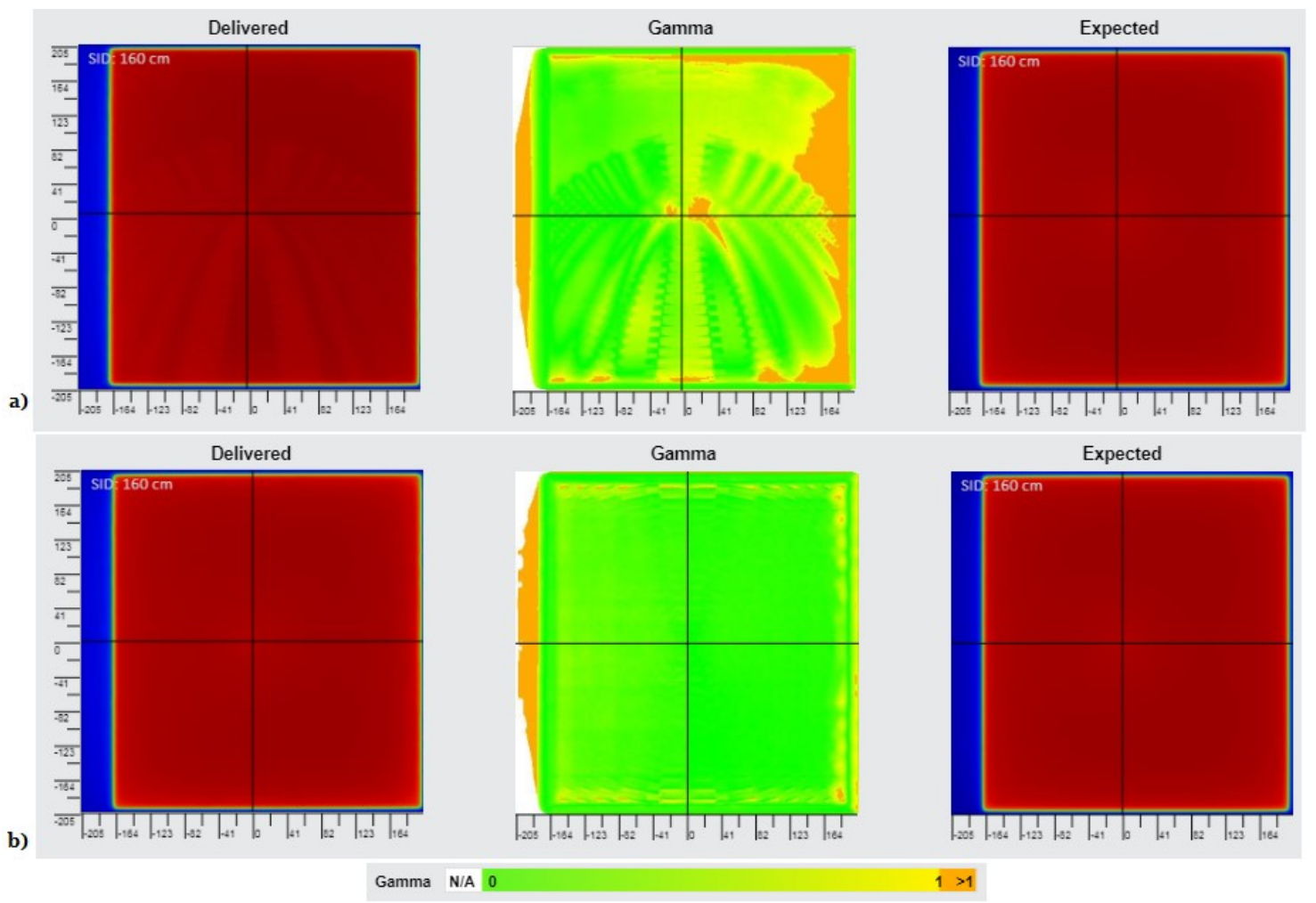

Figure 3. a) An example of the coincidence between the measured (left) portal image and the one predicted by PF (right) during 10MV FZAD Calibration. The Central image shows the distribution of the gamma index value. Orange colored areas correspond to $\gamma(3 \% / 3 \mathrm{~mm}, \mathrm{GN})>1$. b) The result of the comparison after the service calibration of the EPID panel

Table 3. Comparison DoseCHECK and 3DVH by the dose at point and DVH parameters

\begin{tabular}{|c|c|c|c|c|c|c|c|c|}
\hline \multirow[t]{2}{*}{ № } & \multirow[t]{2}{*}{ Localization } & \multirow{2}{*}{ Reference } & \multicolumn{3}{|l|}{$3 \mathrm{DVH}$} & \multicolumn{3}{|c|}{ DoseCHECK } \\
\hline & & & TPS, Gy & QA, Gy & $\Delta, \%$ & TPS, Gy & QA,Gy & $\Delta, \%$ \\
\hline \multirow[t]{4}{*}{1} & \multirow[t]{4}{*}{ Brain } & $\mathrm{D}$ (Isocenter) & 30.91 & 31.13 & -0.74 & 30.91 & 30.92 & 0.03 \\
\hline & & D95(PTV) & 55.81 & $55 \cdot 91$ & 0.18 & 55.81 & $55 \cdot 50$ & -0.56 \\
\hline & & $\operatorname{Dmax}(\mathrm{PTV})$ & 71.29 & 74.17 & 4.04 & 71.29 & 71.02 & -0.38 \\
\hline & & Dmax(Brainstem) & 31.88 & 32.10 & 0.45 & 31.88 & 30.21 & -5.24 \\
\hline \multirow[t]{4}{*}{2} & \multirow[t]{4}{*}{ Head\&Neck } & $\mathrm{D}$ (Isocenter) & 52.38 & 53.64 & -2.40 & 52.38 & 51.2 & -2.25 \\
\hline & & D95(PTV) & 49.46 & 49.40 & -0.24 & 49.46 & 47.90 & -3.15 \\
\hline & & $\operatorname{Dmax}(\mathrm{PTV})$ & 58.27 & 61.90 & 6.18 & 58.27 & 55.66 & -4.48 \\
\hline & & Dmax(Spinal cord) & 28.76 & 28.80 & 0.16 & 28.76 & 26.50 & -7.86 \\
\hline \multirow[t]{5}{*}{3} & \multirow[t]{5}{*}{ Prostate } & $\mathrm{D}$ (Isocenter) & 39.10 & 39.05 & 0.12 & 39.10 & 39.16 & 0.15 \\
\hline & & D95(PTV) & 36.18 & 36.39 & 0.58 & 36.18 & 36.09 & -0.25 \\
\hline & & $\operatorname{Dmax}(\mathrm{PTV})$ & 40.16 & 40.60 & 1.09 & 40.16 & 39.76 & -1.00 \\
\hline & & Dmax(Bladder) & 38.67 & 39.26 & 1.54 & 38.67 & 38.25 & -1.09 \\
\hline & & Dmax(Rectum) & 37.78 & 37.38 & -1.08 & 37.78 & 37.06 & -1.91 \\
\hline \multirow[t]{5}{*}{4} & \multirow[t]{5}{*}{ Lymphoma } & $\mathrm{D}$ (Isocenter) & 38.01 & 37.84 & 0.44 & 38.01 & 36.84 & -3.08 \\
\hline & & D95(PTV) & 35.16 & $35 \cdot 30$ & 0.39 & 35.16 & 33.78 & -3.92 \\
\hline & & $\operatorname{Dmax}(\mathrm{PTV})$ & 40.39 & 42.45 & 5.11 & 40.39 & 39.48 & -2.25 \\
\hline & & Dmean(Lungs) & 7.43 & $7 \cdot 51$ & 1.08 & 7.43 & 7.18 & -3.36 \\
\hline & & D2o(Lungs) & 15.21 & 15.33 & 0.79 & 15.21 & 14.56 & -4.27 \\
\hline
\end{tabular}




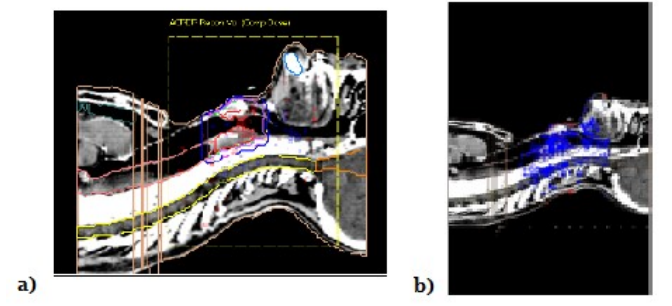

Figure 6. Distribution of "hot"(red) and "cold"(blue) points in patient CT images according to gamma analysis by criteria $\gamma(2 \mathrm{~mm} / 3 \%, \mathrm{GN})$ at $3 \mathrm{DVH}(\mathrm{a})$ and DoseCHECK(b) verification.
Figure 6 shows the distribution of "hot" and "cold" spots on the longitudinal section of the Head\&Neck localization. The presence of a large cluster of spots in DoseCHECK verification indicates a significant reduction in the dose compared to the planned one (Figure 6b). At $3 \mathrm{DVH}$ verification (Figure 6a) there is a local concentration of "hot" spots in the target area.

Figure 7 shows the pre-treatment DVH distributions for Brain localization. The situation with dose distributions confirms the data from Table 3: the calculated values (dotted line) DoseCHECK (right) were below the TPS (solid line) values.
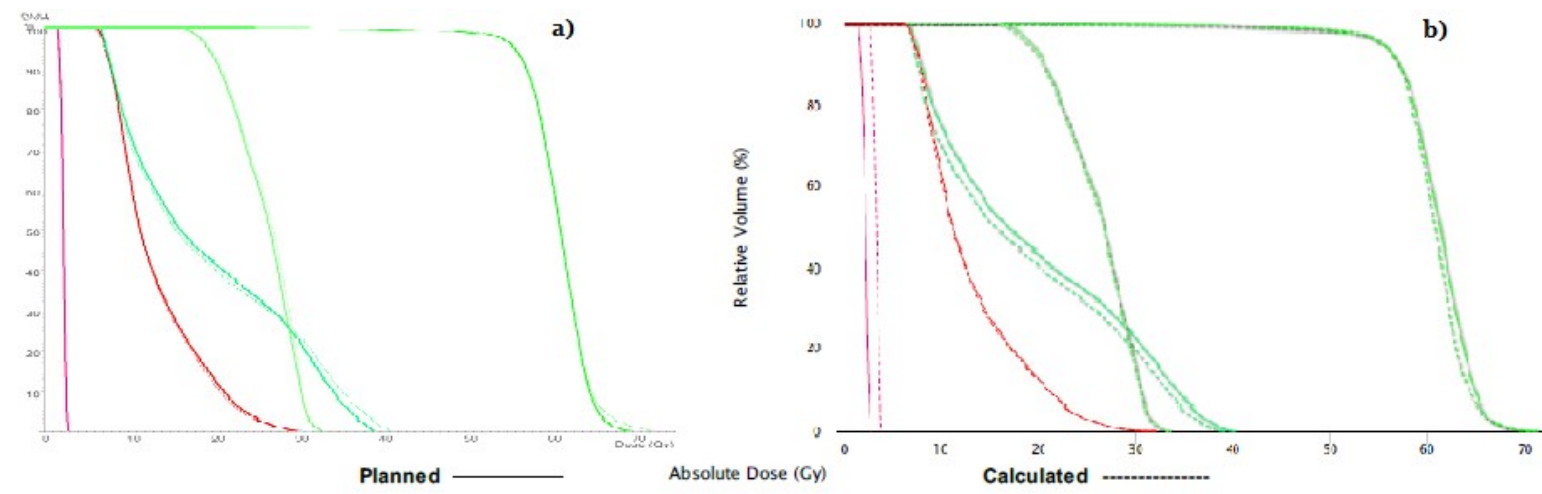

Figure 7. Pre-treatment DVH distribution 3DVD (left) and DoseCHECK (right) for Brain localization

We can see the dose reduction on the target and on the critical organs by comparison of the results of DoseCHECK analysis with the results of the pretreatment QA using the ArcCHECK dosimetric phantom. On the other hand, the EPID-based in vivo dosimetry indicates that QA doses of the prostate case are close to the TPS doses. The reason of that is signalto-dose conversion factors that were adjusted to real linac dose output. We may assume that CCC algorithm with a standard beam model underestimates the dose delivered to the patient. In this regard, we plan to optimize the beam model to the specifics of our TPS in cooperation with the Sun Nuclear Company.

According to the results of the study, we can conclude that linac delivers a higher dose then the prescribed ones. Although the DoseCHECK underestimates the dose distribution, the calculations of dose coverage based on the EPID images in absolute values is higher than only the TPS dose recalculation. This correlates with the results of the $3 \mathrm{D}$ ArcCHECK verification, which also indicates an overdose of most structures (Table 3).

\section{CONCLUSION}

This study describes some aspects of setting up the interaction of the PerFraction system with the elements of the linear accelerator, as well as the need to calibrate the EPID panel in the event of a mismatch of what is delivered and the expected distributions.

The calculation by DoseCHECK based on the standard beam model was also compared to the results of the $3 \mathrm{D}$ phantom-based verification. It is expected that the beam model customization will be carried out in accordance with the TPS model.

After eliminating all the shortcomings and the final implementation of PerFraction in daily practice, it is planned to use this system as a basis for the transition to adaptive radiation therapy.

\section{REFERENCES}

1. H. Zhen, B. E. Nelms, W. A. Tome, "Moving from gamma passing rates to patient DVH-based QA metrics in pretreatment dose QA," Med. Phys., vol. 38, no. 10, pp. $5477-5489$, Oct. 2011.

DOI: $10.1118 / 1.3633904$ PMid: 21992366

2. B. Mijnheer, S. Beddar, J. Izewska, C. Reft, "In vivo dosimetry in external beam radiotherapy," Med. Phys., vol. 40, no. 7, Jul. 2013.

DOI: $10.1118 / 1.4811216$ PMid: 23822404

3. M. Sabet, F. W. Menk, P. B. Greer, "Evaluation of an aSi EPID in direct detection configuration as a waterequivalent dosimeter for transit dosimetry," Med. Phys., vol. 37, no. 4, pp. 1459 - 1467, Apr. 2010. DOI: $10.1118 / 1.3327456$ PMid: 20443467

4. A. Mans et al., "Catching errors with in vivo EPID dosimetry," Med. Phys., vol. 37, no. 6, pp. 2638 - 2644, Jun. 2010.

DOI: $10.1118 / 1.3397807$ PMid: 20632575

5. A. H. Zhuang, A. J. Olch, "Sensitivity study of an automated system for daily patient QA using EPID exit dose images," J. Appl. Clin. Med. Phys., vol. 19, no. 3, pp. $114-124$, May 2018. DOI: $10.1002 / \mathrm{acm} 2.12303$ 
A. V. Vertinskiy et al., In vivo dosimetry system implementation..., RAP Conf. Proc., vol. 4, 2019, 181-186

PMid: 29508529

PMCid: PMC5978566

6. C. Bojechko, M. Phillps, A. Kalet, E. C. Ford, "A quantification of the effectiveness of EPID dosimetry and software-based plan verification systems in detecting incidents in radiotherapy," Med. Phys., vol. 42, no. 9, pp. $5363-5369$, Sep. 2015

DOI: $10.1118 / 1.4928601$ PMid: 26328985

7. I. Olaciregui-Ruiz, R. Rozendaal, B. Mijnheer, A. Mans, "Site-specific alert criteria to detect patient-related errors with 3D EPID transit dosimetry," Med. Phys., vol. 46, no. 1, pp. 45 - 55, Jan. 2019.

DOI: $10.1002 / \mathrm{mp} .13265$

PMid: 30372521

8. S. Bresciani, L. Botez, A. Miranti, M. Stasi, "In Vivo dosimetry using CBCT and EPID device: Analysis of sources of errors in VMAT treatments", Radiother. Oncol., vol. 133, suppl. 1, pp. 249 - 250, Apr. 2019. DOI: 10.1016/So167-8140(19)30904-1
9. R. Jacques, J. Wong, R. Taylor, T. McNutt, "Real-time dose computation: GPU-accelerated source modeling and superposition/convolution," Med. Phys., vol. 38, no. 1, pp. 294 - 305, Jan. 2011.

DOI: $10.1118 / 1.3483785$

PMid: 21361198

10. S. Ahmed et al., "Validation of a GPU-Based 3D dose calculator for modulated beams," J. Appl. Clin. Med. Phys., vol. 18, no. 3, pp. $73-82$, May 2017.

DOI: $10.1002 / \mathrm{acm} 2.12074$

PMid: 28371377

PMCid: PMC5689856

11. EPID Dosimetry in SunCHECK ${ }^{\mathrm{TM}}$ Patient: EPID Calibration, Pre-Treatment $Q A$ and In-Vivo Monitoring, Sun Nucl. Corp., Melbourne (FL), USA.

Retrieved from:

https://www.sunnuclear.com/documents/whitepapers/ EPID-Dosimetry in-SC Patient 021519.pdf

Retrieved on: Dec. 12, 2018 